\title{
The column vector calculus for Thermo Field Dynamics of relativistic quantum fields *
}

\author{
P.A.Henning ${ }^{\dagger}$ \\ Institut für Kernphysik der TH Darmstadt and GSI \\ P.O.Box 110552, D-6100 Darmstadt, Germany \\ May 4, 1993
}

\begin{abstract}
A formalism is discussed which simplifies the calculation of Feynman diagrams at finite temperature.
\end{abstract}

PACS No. 03.70.+k,05.30.-d,05.20.Dd,05.60.+w,05.70.Ln

\footnotetext{
*Work supported by GSI

${ }^{\dagger}$ E-mail address: phenning@tpri6a.gsi.de

$\ddagger$ after July 1st: D-64220
} 


\section{Introduction}

Many efforts in contemporary physics are devoted to the study of quantum systems at finite temperature or density, like e.g. particles propagating in hot, compressed nuclear matter. While the vacuum state of quantum field theory is invariant under Lorentz transformations, matter states have, in general, less symmetry. This leads to a modification of the particle spectrum needed for the description of such a state [1]. For the application of perturbative (i.e. diagrammatic) methods of quantum field theory, this modification has two main effects.

First, one has to use $2 \times 2$ matrix valued propagators, as in Thermo Field Dynamics (TFD) 22] or the Closed-Time-Path formalism [3]. Second, a consistent description requires elementary excitations with a continuous mass spectrum, rather than physical quasi-particles of infinite lifetime [4].

A well defined diagrammatic expansion at finite temperature therefore takes into account a spectral function of "particles" which deviates from a $\delta$-function, i.e., which is more than a mass-shell constraint. In several papers it was shown recently, how the propagators of such an expansion can be diagonalized, and what the physical meaning of the matrix structure is in the framework of Thermo Field Dynamics [5, 6, 7].

In the present work, a calculational recipe for TFD is discussed, which was developed for non-relativistic theories [8]. The method is extended to relativistic quantum fields, where it makes the calculation of Feynman diagrams for matrix valued propagators a very simple task. To this end, a quantum field theory with minimal coupling of fermions and scalar uncharged bosons in thermal equilibrium is considered (see refs. [9, 10 for applications).

In TFD, the thermal instability of observable states is absorbed into a Bogoliubov transformation. This Bogliubov transformation can be written also for interacting systems, where it defines stable, albeit non-observable quasi-particles [5, 6, 7]. At the level of the quasi-particle operators, it can be cast into a single matrix form for the bosonic and the fermionic sector of the model, with a parameter $n$ that resembles the phase-space occupation factor. While one has some freedom in parameterizing the transformation, the most useful way to do so is

$$
\mathcal{B}_{B, F}(n)=\left(\begin{array}{cc}
(1 \pm n) & -n \\
\mp 1 & 1
\end{array}\right)
$$


since it is linear in $n$ [2]. In the fermionic sector, the $n$ are Fermi-Dirac functions, labeled $n_{F}^{+}$for positive energy states and $n_{F}^{-}$for negative energy states. The thermal quasi-particle picture of TFD requires that they depend on a continuous energy variable,

$$
n_{F}^{ \pm}(E)=\frac{f_{F}^{ \pm}(E)}{1+f_{F}^{ \pm}(E)}=\frac{1}{\mathrm{e}^{\beta\left(E \mp \mu_{F}\right)}+1}, \quad f_{F}^{ \pm}(E)=\mathrm{e}^{-\beta\left(E \mp \mu_{F}\right)} .
$$

In the bosonic sector, $n$ is a Bose-Einstein distribution function,

$$
n_{B}(E)=\frac{f_{B}(E)}{1-f_{B}(E)}=\frac{1}{\mathrm{e}^{\beta E}-1}, f_{B}(E)=\mathrm{e}^{-\beta E}
$$

\section{Full propagators in thermal equilibrium}

The full bosonic two-point Green's function, or propagator, at finite temperature has been derived in refs. [6, 7], as

$$
\begin{aligned}
& D^{(a b)}\left(k_{0}, \boldsymbol{k}\right) \quad=\int_{0}^{\infty} d E \rho_{B}(E, \boldsymbol{k}) \times \\
& \left(\left(\mathcal{B}_{B}\left(n_{B}(E)\right)\right)^{-1}\left(\begin{array}{cc}
\frac{1}{k_{0}-E+i \epsilon} & \frac{1}{} \\
& \frac{1}{k_{0}-E-i \epsilon}
\end{array}\right) \mathcal{B}_{B}\left(n_{B}(E)\right) \tau_{3}\right. \\
& \left.-\quad \tau_{3} \mathcal{B}_{B}^{T}\left(n_{B}(E)\right)\left(\begin{array}{cc}
\frac{1}{k_{0}+E-i \epsilon} & \\
& \frac{1}{k_{0}+E+i \epsilon}
\end{array}\right)\left(\mathcal{B}_{B}^{T}\left(n_{B}(E)\right)\right)^{-1}\right) .
\end{aligned}
$$

$\rho_{B}(E, \boldsymbol{k})$ is a positive weight function with support only for positive energy arguments, $\tau_{3}=\operatorname{diag}(1,-1)$.

In the above equation, the terms propagating particle and anti-particle states have been kept separately. Since eqn. (3) can be continued to negative energy arguments, the above propagator simplifies to

$$
\begin{aligned}
D^{(a b)}\left(k_{0}, \boldsymbol{k}\right) & =\int_{-\infty}^{\infty} d E \mathcal{A}_{B}(E, \boldsymbol{k}) \times \\
& \left(\mathcal{B}_{B}\left(n_{B}(E)\right)\right)^{-1}\left(\begin{array}{ll}
\frac{1}{k_{0}-E+i \epsilon} & \frac{1}{k_{0}-E-i \epsilon}
\end{array}\right) \mathcal{B}_{B}\left(n_{B}(E)\right) \tau_{3} .
\end{aligned}
$$


$\mathcal{A}_{B}(E, \boldsymbol{k})$ is the spectral function of the boson field,

$$
\mathcal{A}_{B}(E, \boldsymbol{k})=\rho_{B}(E, \boldsymbol{k}) \Theta(E)-\rho_{B}(-E, \boldsymbol{k}) \Theta(-E),
$$

and the limit of free particles with mass $m$ is recovered when

$$
\mathcal{A}_{B}(E, \boldsymbol{k}) \longrightarrow \operatorname{sign}(E) \delta\left(E^{2}-\boldsymbol{k}^{2}-m^{2}\right) .
$$

The fermion propagator is calculated in a similar way. The occurence of a nonzero chemical potential does not hinder the above simplification, since the occupation number parameters for positive and negative energy states are related by

$$
1-n_{F}^{-}(-E)=n_{F}^{+}(E) \equiv n_{F}(E), \quad\left(f_{F}^{-}(-E)\right)^{-1}=f_{F}^{+}(E) \equiv f_{F}(E) .
$$

Some elementary matrix algebra then gives, in terms of the fermionic spectral function $\mathcal{A}_{F}(E, \boldsymbol{p})$ which has support on the whole real energy axis,

$$
\begin{aligned}
& S_{F}^{(a b)}\left(p_{0}, \boldsymbol{p}\right)=\int_{-\infty}^{\infty} d E \mathcal{A}_{F}(E, \boldsymbol{p}) \times \\
& \tau_{3}\left(\mathcal{B}_{F}\left(n_{F}(E)\right)\right)^{-1}\left(\begin{array}{ll}
\frac{1}{p_{0}-E+i \epsilon} & \frac{1}{p_{0}-E-i \epsilon}
\end{array}\right) \mathcal{B}_{F}\left(n_{F}(E)\right) .
\end{aligned}
$$

The limit of free Dirac particles with mass $M$ is recovered when

$$
\mathcal{A}_{F}(E, \boldsymbol{p}) \longrightarrow\left(p_{\mu} \gamma^{\mu}+M\right) \operatorname{sign}(E) \delta\left(E^{2}-\boldsymbol{p}^{2}-M^{2}\right)
$$

Note, that the above equations for the full propagators contain the full contribution of the negative energy states.

\section{Column vector notation for propagators}

To simplify the use of such matrix valued propagators, a column vector notation was introduced in ref. [8]. It is obtained by rewriting the thermal Bogoliubov matrices defined in eqn. (1) as the outer product of two column vectors according to

$$
\left(\begin{array}{ll}
a_{1} b_{1} & a_{1} b_{2} \\
a_{2} b_{1} & a_{2} b_{2}
\end{array}\right)=\left\{\begin{array}{l}
a_{1} \\
a_{2}
\end{array}\right\}\left\{\begin{array}{l}
b_{1} \\
b_{2}
\end{array}\right\} .
$$


This decomposition works only for matrices with zero determinant. The art in applying this idea therefore lies in splitting the propagator matrices into a sum of terms, each with determinant zero.

For the above propagators this task is trivial: one simply isolates the parts propagating forward and backward in time, this gives the full fermion and boson propagators as

$$
\begin{gathered}
S^{(a b)}\left(p_{0}, \boldsymbol{p}\right)=\int_{-\infty}^{\infty} d E \mathcal{A}_{F}(E, \boldsymbol{p}) n_{F}(E) \times \\
\left(\frac{1}{p_{0}-E+i \epsilon}\left\{\begin{array}{l}
1 \\
1
\end{array}\right\}\left\{\begin{array}{c}
f_{F}^{-1}(E) \\
-1
\end{array}\right\}-\frac{1}{p_{0}-E-i \epsilon}\left\{\begin{array}{c}
-1 \\
f_{F}^{-1}(E)
\end{array}\right\}\left\{\begin{array}{l}
1 \\
1
\end{array}\right\}\right) \\
D^{(a b)}\left(k_{0}, \boldsymbol{k}\right)=\int_{-\infty}^{\infty} d E \mathcal{A}_{B}(E, \boldsymbol{k}) n_{B}(E) \times \\
\left(\frac{1}{k_{0}-E+i \epsilon}\left\{\begin{array}{c}
1 \\
1
\end{array}\right\}\left\{\begin{array}{c}
f_{B}^{-1}(E) \\
1
\end{array}\right\}-\frac{1}{k_{0}-E-i \epsilon}\left\{\begin{array}{c}
1 \\
f_{B}^{-1}(E)
\end{array}\right\}\left\{\begin{array}{l}
1 \\
1
\end{array}\right\}\right)
\end{gathered}
$$

Note, that each column vector is associated with one endpoint of a propagator line. Consequently, an $\mathrm{N}$-point function of the interacting theory is described as an object composed of $N$ column vectors,

$$
\Gamma^{(N)}=\left\{\begin{array}{l}
a_{1} \\
b_{1}
\end{array}\right\}\left\{\begin{array}{l}
a_{2} \\
b_{2}
\end{array}\right\} \cdots\left\{\begin{array}{l}
a_{N} \\
b_{N}
\end{array}\right\} .
$$

Now consider a typical diagram of the perturbative expansion of an interacting quantum field theory. In such a diagram, in general bare vertices and irreducible vertex functions are joined by propagators. The rule for combination of their column vectors now is the following:

Rule 1. If two or more propagator lines, counted by an index $1<l<L$, meet in one $L$-point vertex, and the vertex is still "open", i.e., external lines can be attached to it, combine their column vectors to a new column vector as

$$
\left\{\begin{array}{l}
a_{1} \\
b_{1}
\end{array}\right\} \times\left\{\begin{array}{l}
a_{2} \\
b_{2}
\end{array}\right\} \times \cdots=\left\{\begin{array}{l}
\prod_{l} a_{l} \\
\prod_{l} b_{l}
\end{array}\right\} .
$$


Rule 2. If the vertex is "closed", i.e., if it is an interior vertex and the last possible propagator line with index $L$ is attached to it, contract it to a scalar function

$$
\left\{\begin{array}{l}
a_{L} \\
b_{L}
\end{array}\right\} \bullet\left\{\begin{array}{l}
\prod_{l}^{L-1} a_{l} \\
\prod_{l}^{L-1} b_{l}
\end{array}\right\}=\prod_{l=1}^{L} a_{l}+\prod_{l=1}^{L} b_{l} .
$$

Note, that in ref. 88 a relative negative sign was used in this rule. In the present work, this was absorbed into the definition of the coupling constant, see below.

\section{Applications of the method}

In a first step, the above rules are applied to "one-loop" self energy diagrams. Since the propagators of the model contain the full spectral functions, this "one-loop" approximation can go substantially beyond a naive perturbation theory. First, consider the one-loop polarization tensor,

$$
\Pi^{(a b)}\left(k_{0}, \boldsymbol{k}\right)=-i \int \frac{d^{4} p}{(2 \pi)^{4}} \operatorname{Tr}\left[g^{(a)} S^{(a b)}\left(p_{0}+k_{0}, \boldsymbol{p}+\boldsymbol{k}\right) g^{(b)} S^{(b a)}\left(p_{0}, \boldsymbol{p}\right)\right]
$$

where $a, b$ are the thermal matrix indices taking values 1 and 2 . The coupling constants are (cf. ref. [2]) $g^{1}=-g^{2}=g$, and the exchange of the indices in the second factor is achieved by a simple transposition of the corresponding column vectors. Hence one has

$$
\begin{array}{r}
\Pi^{(a b)}\left(k_{0}, \boldsymbol{k}\right)=-i \int \frac{d^{4} p}{(2 \pi)^{4}} \int_{-\infty}^{\infty} d E d E^{\prime} \operatorname{Tr}\left[\mathcal{A}_{F}(E, \boldsymbol{p}+\boldsymbol{k}) \mathcal{A}_{F}\left(E^{\prime}, \boldsymbol{p}\right)\right] n_{F}(E) n_{F}\left(E^{\prime}\right) \times \\
\left(\frac{1}{p_{0}+k_{0}-E+i \epsilon}\left\{\begin{array}{l}
1 \\
1
\end{array}\right\}\left\{\begin{array}{c}
f_{F}^{-1}(E) \\
-1
\end{array}\right\}-\frac{1}{p_{0}+k_{0}-E-i \epsilon}\left\{\begin{array}{c}
-1 \\
f_{F}^{-1}(E)
\end{array}\right\}\left\{\begin{array}{l}
1 \\
1
\end{array}\right\}\right) \times \\
\left(\frac{1}{p_{0}-E^{\prime}+i \epsilon}\left\{\begin{array}{c}
f_{F}^{-1}\left(E^{\prime}\right) \\
-1
\end{array}\right\}\left\{\begin{array}{l}
1 \\
1
\end{array}\right\}-\frac{1}{p_{0}-E^{\prime}-i \epsilon}\left\{\begin{array}{l}
1 \\
1
\end{array}\right\}\left\{\begin{array}{c}
-1 \\
f_{F}^{-1}\left(E^{\prime}\right)
\end{array}\right\}\right) \times
\end{array}
$$

where the last $\times$ indicates that the product is closed, i.e. the trace also applies to the thermal indices.

In performing the $p_{0}$-integration, out of the four possible products in the integrand only those survive which have different relative sign of $i \epsilon$ in 
their factors. Applying rule 1, the complete polarization tensor therefore is obtained as

$$
\begin{gathered}
\Pi^{(a b)}\left(k_{0}, \boldsymbol{k}\right)=-g^{2} \int \frac{d^{3} \boldsymbol{p}}{(2 \pi)^{3}} \int_{-\infty}^{\infty} d E d E^{\prime} \operatorname{Tr}\left[\mathcal{A}_{F}(E, \boldsymbol{p}+\boldsymbol{k}) \mathcal{A}_{F}\left(E^{\prime}, \boldsymbol{p}\right)\right] n_{F}(E) n_{F}\left(E^{\prime}\right) \times \\
\left(\frac{1}{k_{0}+E^{\prime}-E+i \epsilon}\left\{\begin{array}{c}
-1 \\
1
\end{array}\right\}\left\{\begin{array}{c}
f_{F}^{-1}(E) \\
-f_{F}^{-1}\left(E^{\prime}\right)
\end{array}\right\}-\frac{1}{k_{0}+E^{\prime}-E-i \epsilon}\left\{\begin{array}{c}
f_{F}^{-1}\left(E^{\prime}\right) \\
-f_{F}^{-1}(E)
\end{array}\right\}\left\{\begin{array}{c}
-1 \\
1
\end{array}\right\}\right) .(19
\end{gathered}
$$

In the next step one uses, that this self-energy function appears as the kernel of the Schwinger-Dyson equation for the full boson propagator as

$$
D(k)=D_{0}(k)+D_{0}(k) \bullet \Pi(k) \bullet D(k)
$$

To pick the retarded full propagator out of this sum, keep only the terms with positive $i \epsilon$ in the denominator (the retarded propagator is an analytical function in the upper complex $k_{0}$-plane). Thus, the second term on the r.h.s of this equation has, in the retarded part, the column vector structure

$$
\begin{gathered}
n_{B}\left(k_{0}\right)\left\{\begin{array}{l}
1 \\
1
\end{array}\right\}\left\{\begin{array}{c}
f_{B}^{-1}\left(k_{0}\right) \\
1
\end{array}\right\} \bullet n_{F}(E) n_{F}\left(E^{\prime}\right)\left\{\begin{array}{c}
-1 \\
1
\end{array}\right\}\left\{\begin{array}{c}
f_{F}^{-1}(E) \\
f_{F}^{-1}\left(E^{\prime}\right)
\end{array}\right\} \bullet \\
n_{B}\left(k_{0}\right)\left\{\begin{array}{l}
1 \\
1
\end{array}\right\}\left\{\begin{array}{c}
f_{B}^{-1}\left(k_{0}\right) \\
1
\end{array}\right\}= \\
n_{B}\left(k_{0}\right)\left\{\begin{array}{l}
1 \\
1
\end{array}\right\}\left\{\begin{array}{c}
f_{B}^{-1}\left(k_{0}\right) \\
1
\end{array}\right\}\left(n_{F}\left(E^{\prime}\right)-n_{F}(E)\right)
\end{gathered}
$$

In other words, inserting an arbitrary number of polarization functions retains the same column vector structure as in eqn. (13)! One can thus read off the above equations the expression for the retarded polarization function, which completely determines the retarded full boson propagator, as

$\Pi^{R}\left(k_{0}, \boldsymbol{k}\right)=g^{2} \int \frac{d^{3} \boldsymbol{p}}{(2 \pi)^{3}} \int_{-\infty}^{\infty} d E d E^{\prime} \operatorname{Tr}\left[\mathcal{A}_{F}(E, \boldsymbol{p}+\boldsymbol{k}) \mathcal{A}_{F}\left(E^{\prime}, \boldsymbol{p}\right)\right]\left(\frac{n_{F}\left(E^{\prime}\right)-n_{F}(E)}{k_{0}+E^{\prime}-E+i \epsilon}\right)$.

In ref. [10], this equation was made the basis of an explicit calculation of a bosonic spectral function at finite temperature.

The second example is the Fock diagram,

$$
\Sigma^{(a b)}\left(p_{0}, \boldsymbol{p}\right)=i \int \frac{d^{4} k}{(2 \pi)^{4}} \operatorname{Tr}\left[g^{(a)} S^{(a b)}\left(p_{0}+k_{0}, \boldsymbol{p}+\boldsymbol{k}\right) g^{(b)} D^{(b a)}\left(k_{0}, \boldsymbol{k}\right)\right] .
$$


Following the same steps as above, i.e., contracting the column vector products at both vertices, and performing the $k_{0}$-integration such that only terms with different sign of $i \epsilon$ survive, then gives

$$
\begin{array}{r}
\Sigma^{(a b)}\left(p_{0}, \boldsymbol{p}\right)=g^{2} \int \frac{d^{3} \boldsymbol{k}}{(2 \pi)^{3}} \int_{-\infty}^{\infty} d E d E^{\prime} \mathcal{A}_{F}(E, \boldsymbol{p}+\boldsymbol{k}) \mathcal{A}_{B}\left(E^{\prime}, \boldsymbol{k}\right) n_{F}(E) n_{B}\left(E^{\prime}\right) \times \\
\left(\frac{1}{p_{0}+E^{\prime}-E+i \epsilon}\left\{\begin{array}{c}
1 \\
-1
\end{array}\right\}\left\{\begin{array}{c}
f_{F}^{-1}(E) \\
f_{B}^{-1}\left(E^{\prime}\right)
\end{array}\right\}+\frac{1}{p_{0}+E^{\prime}-E-i \epsilon}\left\{\begin{array}{c}
f_{B}^{-1}\left(E^{\prime}\right) \\
f_{F}^{-1}(E)
\end{array}\right\}\left\{\begin{array}{c}
1 \\
-1
\end{array}\right\}\right)
\end{array}
$$

Again, put this into a Schwinger-Dyson equation

$$
S(p)=S_{0}(p)+S_{0}(p) \bullet \Sigma(p) \bullet S(p),
$$

and isolate the retarded full fermion propagator by keeping only the terms with positive $i \epsilon$ in the denominator. The second term on the r.h.s then has, in the retarded part, the factors

$$
\begin{array}{r}
n_{F}\left(p_{0}\right)\left\{\begin{array}{l}
1 \\
1
\end{array}\right\}\left\{\begin{array}{c}
f_{F}^{-1}\left(p_{0}\right) \\
-1
\end{array}\right\} \bullet n_{F}(E) n_{B}\left(E^{\prime}\right)\left\{\begin{array}{c}
1 \\
-1
\end{array}\right\}\left\{\begin{array}{c}
f_{F}^{-1}(E) \\
f_{B}^{-1}\left(E^{\prime}\right)
\end{array}\right\} \bullet \\
n_{F}\left(p_{0}\right)\left\{\begin{array}{l}
1 \\
1
\end{array}\right\}\left\{\begin{array}{c}
f_{F}^{-1}\left(p_{0}\right) \\
-1
\end{array}\right\}= \\
n_{F}\left(p_{0}\right)\left\{\begin{array}{c}
1 \\
1
\end{array}\right\}\left\{\begin{array}{c}
f_{F}^{-1}\left(p_{0}\right) \\
-1
\end{array}\right\}\left(n_{B}\left(E^{\prime}\right)-n_{F}(E)\right)
\end{array}
$$

Again, the column vector structure of the full propagator (12) is conserved by the insertion of self energy diagrams. From the above expression furthermore follows the retarded self energy function as

$$
\Sigma^{R}\left(p_{0}, \boldsymbol{p}\right)=g^{2} \int \frac{d^{3} \boldsymbol{k}}{(2 \pi)^{3}} \int_{-\infty}^{\infty} d E d E^{\prime} \mathcal{A}_{F}(E, \boldsymbol{p}+\boldsymbol{k}) \mathcal{A}_{B}\left(E^{\prime}, \boldsymbol{k}\right)\left(\frac{n_{B}\left(E^{\prime}\right)-n_{F}(E)}{p_{0}+E^{\prime}-E+i \epsilon}\right) .
$$

\section{Conclusion}

In the present work, the column vector calculus of Thermo Field Dynamics was introduced for relativistic quantum fields. The simplicity of its application was shown in two examples: two well known equations for retarded self energy functions have been obtained within a few lines. 
While this might not be a great gain for the generalized one-loop examples given here, it makes higher order calculations much simpler than with other methods. An example for a non-relativistic vertex correction is dicussed in ref. [8]. The conclusion is, that the column vector calculus is even simpler to apply than the Matsubara technique.

In ref. [10], the above eqn. (22) was used for an application of TFD. There we considered a model with two different types of fermions, i.e., nucleons and $\Delta_{33}$ resonances, coupled to a pion field, and calculated the pionic spectral function. More sophisticated applications are in progress. 


\section{References}

[1] H.J.Borchers, R.N.Sen, Commun.Math.Phys.21 (1975) 101

[2] H.Umezawa, Advanced Field Theory: Micro, Macro and Thermal Physics, (American Institute of Physics, 1993)

[3] J.E.Davis and R.J.Perry, Phys.Rev.C 43 (1991) 1893

[4] N.P.Landsman, Ann.Phys. 186 (1988) 141

[5] Y.Yamanaka, H.Umezawa, K.Nakamura and T. Arimitsu, Thermo Field Dynamics in Time Representation, University of Alberta Preprint (1992)

[6] P.A.Henning and H.Umezawa, Diagonalization of Propagators in TFD for Relativistic Quantum Fields GSI-Preprint 92-61 (1992), subm. to Nucl.Phys. B

[7] P.A.Henning and H.Umezawa, Phys.Lett. B 303 (1993) 209

[8] H.Chu and H.Umezawa

Time ordering theorem and calculational recipes for TFD;

Phys.Lett. A (1993) in press

[9] P.A.Henning, Nucl.Phys. A546 (1992) 653

[10] P.A.Henning and H.Umezawa, The Delta-hole model at finite temperature

GSI-Preprint 93-23 (1993), subm. to Nucl.Phys. A 